\title{
Robust and optimal control
}

\author{
John Doyle ${ }^{1}$ \\ Control and Dynamical Systems \\ California Institute of Technology \\ Pasadena, CA 91125 \\ doyle@hot.caltech.edu \\ http://hot.caltech.edu/ doyle
}

\begin{abstract}
This paper will very briefly review the history of the relationship between modern optimal control and robust control. The latter is commonly viewed as having arisen in reaction to certain perceived inadequacies of the former. More recently, the distinction has effectively disappeared. Once-controversial notions of robust control have become thoroughly mainstream, and optimal control methods permeate robust control theory. This has been especially true in H-infinity theory, the primary focus of this paper, which will serve as a short introduction to the talk. Much of this is taken directly from [33], which also has most of the references.
\end{abstract}

\section{The 70's, briefly}

We could trace the origins of robust control almost arbitrarily far back in time, since robustness has always been the point of feedback, but I'll start this narrative more recently by quickly recalling the controversy about LQG robustness in the mid to late 70's. One of the foundations of modern control theory was optimal control, which was tremendously successful in a variety of applications. The modern optimal control paradigm for feedback design, the LQG problem, however, had relatively little impact on practical control design. One of the critiques of LQG was that it did not directly address many issues that were already well-understood in at least some limited way in classical control, and gain and phase margins were often pointed to as an example of this. The LQG proponents could, however, point to certain guaranteed properties of LQ regulators as indications of inherent robustness. As we now know, these guarantees were of no practical value, and indeed the whole notion of guaranteed margins is a bit silly. But that's another paper....

At about the same time, singular values or the $\mathcal{H}_{\infty}$ norm was proposed for robustness analysis of multivariable systems. This point of view added necessity

\footnotetext{
${ }^{1}$ The author gratefully acknowledges the support of AFOSR.

to the small gain methods of the 1960 s $[30,29,23]$. That is, whereas small gain gave sufficient conditions for stability for a set of uncertainty, the robust control interpretation was that the same condition was necessary and sufficient for a particular set. This emphasis on necessity motivated much study of the potential conservativeness of robustness measures and techniques for reducing it.

One of the motivations for the original introduction of $\mathcal{H}_{\infty}$ methods by Zames [31] was to emphasize plant uncertainty. The $\mathcal{H}_{\infty}$ norm was found to be appropriate for specifying both the level of plant uncertainty and the signal gain from disturbance inputs to error outputs in the controlled system. The $\mathcal{H}_{\infty}$ norm gives the maximum energy gain (the induced $\mathcal{L}_{2}$ system gain), or sinusoidal gain of the system. This is in contrast to the $\mathcal{H}_{2}$ norm, which gives the variance of the output given white noise disturbances. The robust stability consequence was the main motivation for the development of $\mathcal{H}_{\infty}$ methods rather than the worst case signal gain. We compromised on performance to get one norm that let us do everything. With this compromise, we could then talk about robust performance with structured uncertainty.

\section{The 80's and the rise of $\mathcal{H}_{\infty}$}

The synthesis of controllers that achieve an $\mathcal{H}_{\infty}$ norm specification gives a well-defined mathematical problem whose solution became a major research focus in the $1980 \mathrm{~s}$. Most of the original solution techniques were in an input-output setting and involved analytic functions (Nevanlinna-Pick interpolation) or operator-theoretic methods (Sarason, AAK, Ball-Helton) and such derivations involved a fruitful collaboration between operator theorists and control engineers. Indeed, $\mathcal{H}_{\infty}$ theory seemed to many to signal the beginning of the end for the state-space methods which had dominated control for the previous 20 years. Unfortunately, the standard frequency-domain approaches to $\mathcal{H}_{\infty}$ started running into significant obstacles in dealing with multi-input multi-output (MIMO) systems, both mathematically 
and computationally, much as the $\mathcal{H}_{2}$ (or LQG) theory of the 1950's had.

Not surprisingly, the first solution to a general rational MIMO $\mathcal{H}_{\infty}$ optimal control problem [2], which we will refer to as the 1984 approach, relied heavily on state-space methods, although more as a computational tool than in any essential way. The procedure involved state-space inner/outer and coprime factorizations of transfer function matrices which reduced the problem to a Nehari/Hankel norm problem solvable by the state-space method in Glover [1984]. Both $[6,7]$ give expositions of this approach, which in a mathematical sense "solved" the general rational problem. Much of the subsequent work in $\mathcal{H}_{\infty}$ control theory focused on the $2 \times 2$-block problem that was a central part of this solution, either in the model-matching or general distance forms. Unfortunately, the associated complexity of computation was substantial, involving several Riccati equations of increasing dimension, and formulae for the resulting controllers tended to be very complicated and have high state dimension. Encouragement came from $[14,15]$ who showed, for problems transformable to $2 \times 1$-block problems, that a subsequent minimal realization of the controller has state dimension no greater than that of the plant. This suggested the likely existence of similarly low dimension optimal controllers in the general $2 \times 2$ case. Additional progress came from Ball and Cohen, Jonckheere and Juang, Foias and Tannenbaum, Hung, Kwakernaak, and Kimura.

Simple state space $\mathcal{H}_{\infty}$ controller formulae were first announced in Glover and Doyle [8] (after some sustained manipulation). However the very simplicity of the new formulae and their similarity with the $\mathcal{H}_{2}$ ones suggested a more direct approach. Independent encouragement for a simpler approach to the $\mathcal{H}_{\infty}$ problem came from papers by Khargonekar, Petersen, Rotea, and Zhou [11, 12]. They showed that for the statefeedback $\mathcal{H}_{\infty}$ problem one can choose a constant gain as a (sub)optimal controller. In addition, a formula for the state-feedback gain matrix was given in terms of an algebraic Riccati equation. Also, these papers established connections between $\mathcal{H}_{\infty}$-optimal control, quadratic stabilization, and linear-quadratic differential games. They showed that the state-feedback $\mathcal{H}_{\infty}$ problem can be solved by solving an algebraic Riccati equation and completing the square.

Derivations of the controller formulae in Glover and Doyle using derivations more akin to the above state feedback results were given in Doyle, Glover, Khargonekar and Francis [3]. In addition to providing controller formulae that are simple and expressed in terms of plant data as in [8], the methods in that paper are a fundamental departure from the 1984 approach. In particular, the Youla parameterization and the resulting model-matching problem of the 1984 solution are avoided entirely; replaced by a more purely state-space approach involving observer-based compensators a pair of $2 \times 1$ block problems, and a separation argument. The operator theory still plays a central role (as does Redheffer's work on linear fractional transformations), but its use is more straightforward. The key to this was a return to simple and familiar state-space and optimal control tools, in the style of Willems [28], such as completing the squares and Riccati equations. Interestingly, linear matrix inequalities (LMIs) were also mentioned in [28], and they have subsequently taken on a much bigger role in robust control.

Relations between $\mathcal{H}_{\infty}$ have now been established with many other topics in modern control: e.g. risk sensitive control of Whittle [1981, 1990]; differential games (see Başar and Bernhard [1991], Limebeer et al [1992], Green and Limebeer [1995]); J-lossless factorization (Green [1992]); maximum entropy methods of Dym and Gohberg [1986] (see Mustafa and Glover [1990]). The state-space theory of $\mathcal{H}_{\infty}$ has been also been carried much further, by generalizing time-invariant to timevarying, infinite horizon to finite horizon, and finite dimensional to infinite dimensional and even to some nonlinear results. Most of these results used fairly conventional modern optimal control techniques.

\section{Robust LQG at last?}

The operator-theoretic methods of the early $80 \mathrm{~s}$ had not only signaled a departure from the state-space techniques of modern control, but also broken with a long tradition in regard to the modeling of noise. From communication theory to Kalman filtering, noise has been modeled in the context of stochastic theory, which has gained wide acceptance since it naturally incorporates the broadband spectral structure of most real-world disturbances, leading to performance specifications in terms of RMS values of the frequency response $\left(\mathcal{H}_{2}\right.$ norms). In contrast, $\mathcal{H}_{\infty}$ control had to offer a worstcase point of view in which an adversarial noise would excite the peak frequency, which would seem misguided were it not for the fact that it allowed for a common treatment of noise with the often prevalent dynamic uncertainty.

As state-space $\mathcal{H}_{\infty}$ theory matured in the late 80 s and brought the focus back to modern control techniques, the possibility of restoring the $\mathcal{H}_{2}$ treatment of noise, in an $\mathcal{H}_{\infty}$ uncertainty context, seemed to be again within reach. A number of versions of this mixed $\mathcal{H}_{2} / \mathcal{H}_{\infty}$ control problem appeared (a few are $[1,13,32,4,21,25]$ ), the ultimate objective being the question of robust $\mathcal{H}_{2}$ performance: rejection of white signals in the worstcase over a set of plants. Significant progress on this problem is reported in $[26,22,32,10,5]$, deriving 
bounds for the worst-case $\mathcal{H}_{2}$ cost based on state-space techniques. Still, the $\mathcal{H}_{\infty}$ and $\mu$ frameworks retained some advantage, with the availability of convex frequency domain tests for robust performance, and the theoretical characterization of such conditions as necessary and sufficient robustness tests [24, 16, 27]. Such strong results seemed out of reach for the a problem which had to combine stochastic and worst-case techniques.

It turns out, however, that the set-based methodology can indeed be extended to the modeling of white noise, as developed recently in Paganini's thesis [17]. By employing statistical tests in order to identify a typical noise set, robust $\mathcal{H}_{2}$ performance can be viewed as a constrained problem in a worst-case setting [18]. Extending the scope of the quadratic constraint methodology, a frequency domain convex condition for robustness analysis is derived $[20,19]$, which for the first time places it on an equal footing with the $\mathcal{H}_{\infty}$ performance measure; with these results, the cycle of research which originated in the LQG robustness theory can be considered essentially closed. While some synthesis questions still remain, it is clear that the main tools are in place for addressing such questions.

\section{OK, but what about real robust control?}

This paper has focused on $\mathcal{H}_{\infty}$ theory and its relationship with optimal. control. What about $L_{1}, \mu$, real parameters, the rise and fall of vertex and edge theorems, NP-hardness, robust modeling and identification, etc.? These are all equally interesting and important topics, but have turned out to have less direct connections with optimal control than has $\mathcal{H}_{\infty}$ theory.

\section{References}

[1] Bernstein, D.S. and W.M. Haddad (1989). "LQG control with an $\mathcal{H}_{\infty}$ performance bound: A Riccati equation approach," IEEE Trans. Auto. Control, vol. AC-34, pp. 293-305.

[2] Doyle, J. C. (1984). "Lecture notes in advances in multivariable control," ONR/Honeywell Workshop, Minneapolis.

[3] Doyle, J.C., K. Glover, P.P. Khargonekar, B.A. Francis (1989). "State-space solutions to standard $\mathcal{H}_{2}$ and $\mathcal{H}_{\infty}$ control problems," IEEE Trans. Auto. Control, vol. AC-34, No. 8, pp. 831-847. Also see 1988 American Control Conference, Atlanta, June, 1988.

[4] Doyle, J. C., K. Zhou, K. Glover, and B. Bodenheimer (1994). "Mixed $\mathcal{H}_{2}$ and $\mathcal{H}_{\infty}$ performance objectives II: optimal control," IEEE Trans. on Automat. Contr., vol. 39, no. 8, pp. 1575-1587.
[5] Feron E., "Analysis of Robust $\mathcal{H}_{2}$ Performance with Multipliers", Proc. 1994 CDC, Orlando, FA. pp. 2015-2021.

[6] Francis, B. A. (1987). A course in $\mathcal{H}_{\infty}$ control theory, Lecture Notes in Control and Information Sciences, vol. 88 .

[7] Francis, B.A. and J.C. Doyle (1987). "Linear control theory with an $\mathcal{H}_{\infty}$ optimality criterion," SIAM J. Control Opt., vol. 25, pp. 815-844.

[8] Glover, K. and J. Doyle (1988). "State-space formulae for all stabilizing controllers that satisfy an $\mathcal{H}_{\infty}$ norm bound and relations to risk sensitivity," Systems and Control Letters, vol. 11, pp. 167-172.

[9] Glover, K. and J. C. Doyle (1989). "A state space approach to $\mathcal{H}_{\infty}$ optimal control," in Three Decades of Mathematical Systems Theory: A Collection of Surveys at the Occasion of the 50th Birthday of Jan $C$. Willems, H. Nijmeijer and J. M. Schumacher (Eds.), Springer-Verlag, Lecture Notes in Control and Information Sciences, vol. 135, 1989.

[10] Hall S., How J., " Mixed $\mathcal{H}_{2} / \mu$ Performance Bounds using Dissipation Theory", 1993 CDC, San Antonio,1536-1541.

[11] Khargonekar, P.P., I.R. Petersen, and M.A. Rotea (1988). " $\mathcal{H}_{\infty}$ - optimal control with statefeedback," IEEE Trans. on Auto. Control, vol. AC-33, pp. 786-788.

[12] Khargonekar, P.P., I.R. Petersen, and K. Zhou (1990). "Robust stabilization and $\mathcal{H}_{\infty}$-optimal control," IEEE Trans. Auto. Contr., Vol. 35, No. 3, pp. $356 \sim 361$.

[13] Khargonekar, P. P. and M. A. Rotea (1991). "Mixed $\mathcal{H}_{2} \mathcal{H}_{\infty}$ control: a convex optimization approach," IEEE Trans. Automat. Contr., vol. 36, no. 7, pp. 824-737.

[14] Limebeer, D.J.N. and G.D. Halikias (1988). "A controller degree bound for $\mathcal{H}_{\infty}$-optimal control problems of the second kind," SIAM J. Control Opt., vol. 26 , no. 3 , pp. 646-677.

[15] Limebeer, D.J.N. and Y.S. Hung (1987). "An analysis of pole-zero cancellations in $\mathcal{H}_{\infty}$-optimal control problems of the first kind," SIAM J. Control Opt., vol.25, pp. 1457-1493.

[16] Megretski A., Treil S., " Power Distribution Inequalities in Optimization and Robustness of Uncertain Systems", Journal of Mathematical Systems, Estimation and Control, Vol 3, No.3, pp. 301-319, 1993.

[17] Paganini F., Sets and Constraints in the Analysis of Uncertain Systems, $\mathrm{PhD}$ Thesis, California Institute of Technology, 1996.

[18] Paganini F., " A Set-Based Methodology for White Noise Modeling", IEEE Trans. Automat. Contr., to appear October 1996. 
[19] Paganini F., " Robust $\mathcal{H}_{2}$ Analysis for Continuous Time Systems" Tech Report LIDS-P-2342, Massachusetts Institute of Technology, 1996.

[20] Paganini F., " Robust $\mathcal{H}_{2}$ Performance: Guaranteeing Margins for LQG Regulators", Tech. Rep. CDS95-031, California Institute of Technology, submitted to IEEE Trans. A.C.

[21] Peters M.A., and Stoorvogel A.A., " Mixed $\mathcal{H}_{2} / \mathcal{H}_{\infty}$ Control in a Stochastic Framework", Lin. Alg. \& Apps., Vol 205-206, pp. 971-996, 1994.

[22] Petersen I., McFarlane D.,Rotea M., " Optimal guaranteed cost control of discrete-time uncertain linear systems", 1993 IFAC World Congress, Sydney, pp.407-410.

[23] Safonov, M.G. (1980). Stability and robustness of multivariable feedback systems, MIT Press.

[24] Shamma, J., "Robust Stability with Time Varying Structured Uncertainty", IEEE TAC, 39, 4, pp 714$724,1994$.

[25] Sznaier M., "An (Almost) Exact Solution to General SISO Mixed $\mathcal{H}_{2} / \mathcal{H}_{\infty}$ Problems via Convex Optimization", Proceedings 1993 ACC, San Francisco, CA, pp. 250-254.

[26] Stoorvogel A.A., " The Robust $\mathcal{H}_{2}$ Control Problem: A Worst-Case Design", IEEE TAC, 38, 9, 1358$1370,1993$.

[27] Poolla K., Tikku A., " Robust Performance against Time-Varying Structured Perturbations", IEEE Trans. AC., Vol 40, 9, pp. 1589-1602, 1995.

[28] Willems, J.C. (1971). "Least Squares Stationary Optimal Control and the Algebraic Riccati Equation", IEEE Trans. Auto. Control, vol. AC-16, no. 6, pp. 621634.

[29] Willems, J.C. (1971). The analysis of feedback systems, MIT Press.

[30] Zames, G. (1966). "On the input-output stability of nonlinear time-varying feedback systems, parts I and II," IEEE Trans. Auto. Control, vol. AC-11, pp. 228 and 465 .

[31] Zames, G. (1981). "Feedback and optimal sensitivity: model reference transformations, multiplicative seminorms, and approximate inverses," IEEE Trans. Auto. Control, vol. AC-26, pp. 301-320.

[32] Zhou, K., K. Glover, B. Bodenheimer, and J. Doyle (1994). "Mixed $\mathcal{H}_{2}$ and $\mathcal{H}_{\infty}$ performance objectives I: robust performance analysis," IEEE Trans. on Automat. Contr., Vol. 39, No. 8, pp. 1564-1574.

[33] Zhou, K., J. C. Doyle, K. Glover. Robust and optimal control, Prentice Hall. 